\title{
Patient benefit of dog-assisted interventions in health care: a systematic review
}

\author{
Martina Lundqvist ${ }^{*}$, Per Carlsson ${ }^{1}$, Rune Sjödahl ${ }^{2}$, Elvar Theodorsson ${ }^{3}$ and Lars-Åke Levin ${ }^{1}$
}

\begin{abstract}
Background: Dogs are the most common companion animal, and therefore not surprisingly a popular choice for animal-assisted interventions. Dog-assisted interventions are increasingly used in healthcare. The aim of the review was to conduct a systematic literature review of quantitative studies on dog-assisted interventions in healthcare, with the intention of assessing the effects and cost-effectiveness of the interventions for different categories of patients.
\end{abstract}

Methods: A systematic review of the scientific literature reporting results of studies in healthcare, nursing home or home care settings, was conducted. The inclusion criteria applied for this review were: quantitative studies, inclusion of at least 20 study subjects, existence of a control and performed in healthcare settings including nursing homes and home care. The electronic databases PubMed, AMED, CINAHL and Scopus were searched from their inception date through January 2017, for published articles from peer-reviewed journals with full text in English.

Results: Eighteen studies that fulfilled the inclusion criteria, and were judged to be of at least moderate quality, were included in the analysis. Three of them showed no effect. Fifteen showed at least one significant positive effect but in most studied outcome measures there was no significant treatment effect. Dog-assisted therapy had the greatest potential in treatment of psychiatric disorders among both young and adult patients. Dog-assisted activities had some positive effects on health, wellbeing, depression and quality of life for patients with severe cognitive disorders. Dog-assisted support had positive effects on stress and mood.

Conclusions: The overall assessment of the included studies indicates minor to moderate effects of dog-assisted therapy in psychiatric conditions, as well as for dog-assisted activities in cognitive disorders and for dog-assisted support in different types of medical interventions. However, the majority of studied outcome measures showed no significant effect.

Keywords: Animal-assisted therapy, Cost-benefit analysis, Dogs, Dog-assisted activity, Dog-assisted intervention, Dog-assisted support, Dog-assisted therapy, Outcome assessment (health care), Quality of life, Treatment outcome

\section{Background}

Interaction with animals has been a favorite human pursuit since the dawn of history. Numerous studies have reported that animals exert favorable effects on psychological, physiological and social aspects of human wellbeing [1]. The increasing use of animals in health and social care is therefore not surprising.

\footnotetext{
* Correspondence: martina.lundqvist@liu.se

'Department of Medical and Health Sciences, Division of Health Care

Analysis, Linköping University, 581 83, Linköping, Sweden

Full list of author information is available at the end of the article
}

Animal-Assisted Interventions (AAI) are more or less goal oriented and structured interventions that intentionally incorporate animals in health, education and human service for the purpose of therapeutic gains and improved health and wellness [2]. AAI usually consists of three subcategories; Animal-Assisted Therapy (AAT), AnimalAssisted Education (AAE) and Animal-Assisted Activities (AAA). These concepts are defined in a slightly different way by various organizations. According to the International Association of Human-Animal Interaction Organizations (IAHAIO), AAT is a goal oriented, planned and 
structured therapeutic intervention directed and/or delivered by health, education and human service professionals, and intervention progress is measured and included in professional documentation [3]. According to the American Veterinary Medical Association (AVMA), the definition AAT should also be an integral part of the treatment process [4]. Most definitions of AAA differ from AAT in several aspects; the absence of specific treatment goals, delivery by volunteers, spontaneous visit content and no obligation to document. According to the IAHAIO definition, AAA is a "planned and goal oriented informal interaction and visitation conducted by the human-animal team for motivational, educational and recreational purposes. Human-animal teams must have received at least introductory training, preparation and assessment to participate in informal visitations." [3]. Another difference between AAT and AAA seems to be the purpose, which is more focused on wellbeing in AAA and on health improvement in AAT. We also understand that AAA could be provided by different degrees of integration with a formal treatment process. However, this leads us to conclude that there are no established definitions and that there is no clear division between AAT and AAA.

Dogs are the most common companion animal and therefore not surprisingly a popular choice for AAI [1]. They are keen observers of human reactions through their exceptional ability to read signs of will and emotion from human faces [5]. They also exhibit a behavior that humans interpret as happy, friendly and affectionate which makes them suitable to be used in interventions with a therapeutic aim [1]. This review is therefore delimited to interventions with dogs as the assisting animal, transposing the term AAT to the corresponding Dog-Assisted Therapy (DAT) and AAA to Dog-Assisted Activity (DAA).

In our review we have identified another relevant subcategory to Dog-Assisted Interventions (DAI), namely Dog-Assisted Support (DAS). DAS is delivered by a trained health care professional or a volunteer, within the scope of the professionals' practice, in order to have alleviating or distracting effects during short term diagnostic or therapeutic interventions. In our opinion, DAS deserves to be a separate category since DAS in contrast to DAA has a specific diagnostic or therapeutic intention, especially in reducing anxiety and stress both in diagnostic and therapeutic contexts, but compared to DAT, DAS has no specific therapeutic goal. Rather, DAS focuses on reduced anxiety and quality of life with indirect effect to facilitate a regular treatment process. Figure 1 illustrates our identified categories.

Previous reviews of DAI studies [6-10] have focused exclusively on single conditions or on specific populations. In this review we have included various conditions without any limitation of the characteristics of the population, except for sample size.

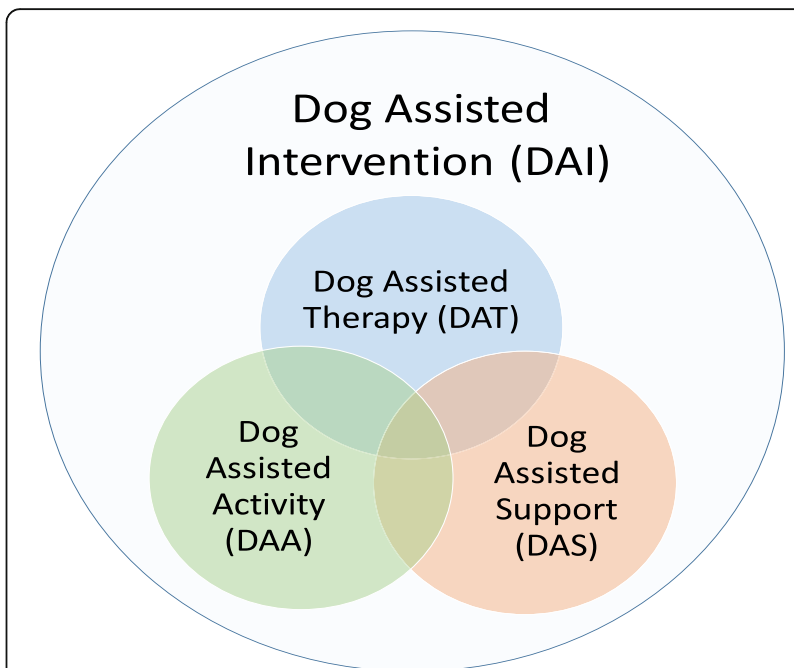

Fig. 1 Dog-assisted intervention (DAl) comprises dog-assisted therapy (DAT), dog-assisted activity (DAA) or dog-assisted support (DAS)

\section{Aim}

The aim of the review was to conduct a systematic literature review of quantitative studies on dog-assisted interventions in healthcare, with the intention of assessing the effects and cost-effectiveness of the interventions for different categories of patients.

\section{Method}

\section{Search methods for identification of studies}

Studies were identified by searching the electronic databases PubMed, AMED, CINAHL and Scopus from their inception date through January 2017. Some of the included studies were identified outside standard database searches (e.g. hand searching reference lists from included articles and systematic reviews).

We searched for various terms of DAI and outcomes. The search was conducted as presented in Table 1.

\section{Selection of studies}

The inclusion criteria applied in the review were determined before the initial literature search. The first four

Table 1 Search strategy

Dog OR canine
AND
Animal-assist* OR Dog-assist* OR Pet-assist* OR Canine-assist* OR
animal-therap* OR dog-therap* OR pet-therap* OR canine-therap* OR
"animal visitation" OR "dog visitation" OR "pet visitation" OR "canine
visitation" OR animal-physiotherap* OR dog-physiotherap* OR
pet-physiotherap* OR canine-physiotherap* OR therapy-dog OR
visiting-dog
AND

Effect $O R$ effectiveness $O R$ benefit $O R$ quality of life OR economics $O R$ cost-effectiveness OR ethics OR outcome 
criteria were a consequence of the scope of the study. The last three criteria were determined by the research group as basic quality criteria to be fulfilled. The inclusion criteria;

- Studies of DAI performed in healthcare settings including nursing homes and home care.

- Studies collecting primary data.

- Quantitative studies (i.e. measuring outcomes with numerical scales).

- Separate result presentation of effects.

- At least 20 study subjects.

- Existence of a control.

- Published articles from peer-reviewed journals with full text in English.

One of the authors (ML) conducted the initial search in May 2016, as well as a second search in September 2016 and a third search in January 2017. All the titles and abstracts from the identified studies were examined to determine the relevance of the articles. If the title and abstract met with the inclusion criteria the study passed on to the next stage of the review process. Studies with insufficient information in title and abstract were also included in the next stage of the review process. The studies that remained after the initial selection, were read in full to confirm eligibility and determine quality.

\section{Review of quality}

Pairs of authors independently read the articles in full to assess eligibility and to determine the quality. Traditional criteria for judgment of scientific quality were used, including; adequate control group(s), control of confounders, randomization, adequately described experimental design, and relevant measured outcome variables [11, 12]. The quality of the studies was rated as high, moderate or low. A study determined as being of high quality had to fulfill all the above mentioned criteria and a study determined as being of moderate quality had to fulfill most of the above mentioned criteria. When a study was determined as being of low quality it failed to fulfill several of the above mentioned criteria or had major shortcomings in some of the criteria. Studies rated as moderate or high were included in the analysis. Studies rated as low quality were excluded, but are presented with a comment in an additional file (see Additional file 1). For the included studies the following data was extracted and gathered into a structured table; reference, characteristics of patients (age, gender, condition), type of study, type of intervention, study period, sample size, outcomes, author conclusion, and scientific quality rating.

\section{Categorization}

To establish in what context DAIs have effect we categorized the interventions as therapeutic (DAT), activating
(DAA) or supportive (DAS) intervention, based on the criteria presented in Table 2.

\section{Results \\ Results of the search}

The result of the search is illustrated in the flow chart in Fig. 2. It resulted in 1445 unique articles (after duplicate removal). Another 28 studies were included through other sources. Based on the information in the title and the abstract 1402 articles did not meet the eligibility criteria and were therefore excluded. The main reason for early exclusion was that the article did not scope DAI. Additionally, 53 articles were excluded after reading the full text. Of these 53 articles, 28 were excluded since they did not meet the inclusion criteria and 25 were excluded due to low quality [13-37] (see Additional file 1). Finally, 18 studies that fulfilled the inclusion criteria, and were judged to be of at least moderate quality, were included in the final analysis. None of the studies were considered to be of high quality. A summary of the 18 studies included in the final analysis is presented in Tables 3 and 4.

\section{Included studies}

The included studies were published in the time period 1997-2016. Countries of the corresponding authors that were represented were USA (8 studies), Italy (4 studies), Australia, Columbia, Denmark, Germany, Norway, Spain (1 study each).

Seventy-eight percent of the studies were randomized controlled trials. Two studies were cohort studies with a pre-post intervention. One study adopted a withinsubject, time series design and one used a crossover design.

\section{Studied patients}

The number of patients included in the studies varied, between 23 and 100. Eleven studies had 20-40, four had 41-60, two had 61-80, and one had 81-100 patients (Table 3). In 11 of the 18 studies the patients were adults. The remaining studies included children and adolescents. The gender distributions favored women, 14 of the 18 studies had $>50 \%$ women.

\section{Studied disorders}

Studies on cognitive disorders dominated the selected studies [38-44]. In four studies the patient conditions were psychiatric disorders, of which three included children or adolescents with psychiatric disorders and one included patients cared for in institutions due to psychiatric disorders [45-48]. Six studies examined stress and mood [49-54]. Target symptoms and diseases were children undergoing physical examination, children undergoing dental procedure, children undergoing venipuncture, patients with cancer and older adults. In 
Table 2 Criteria for categorization of interventions

\begin{tabular}{llll}
\hline & DAT & DAA & DAS \\
\hline Intervention & & & No specific goals \\
Aim of action & Goal orientated & No specific goals & Spontaneous \\
Content of activity & Structured & Spontaneous & Single session \\
Amount of activity & Multiple sessions & Multiple sessions & High \\
Treatment integration & High & Low & Volunteer \\
Dog handler/therapist & Educated & Volunteer & Semi-professional \\
& Professional & Not professional & Distraction \\
Effect/Focus & Health & Well-being & Stress reduction
\end{tabular}

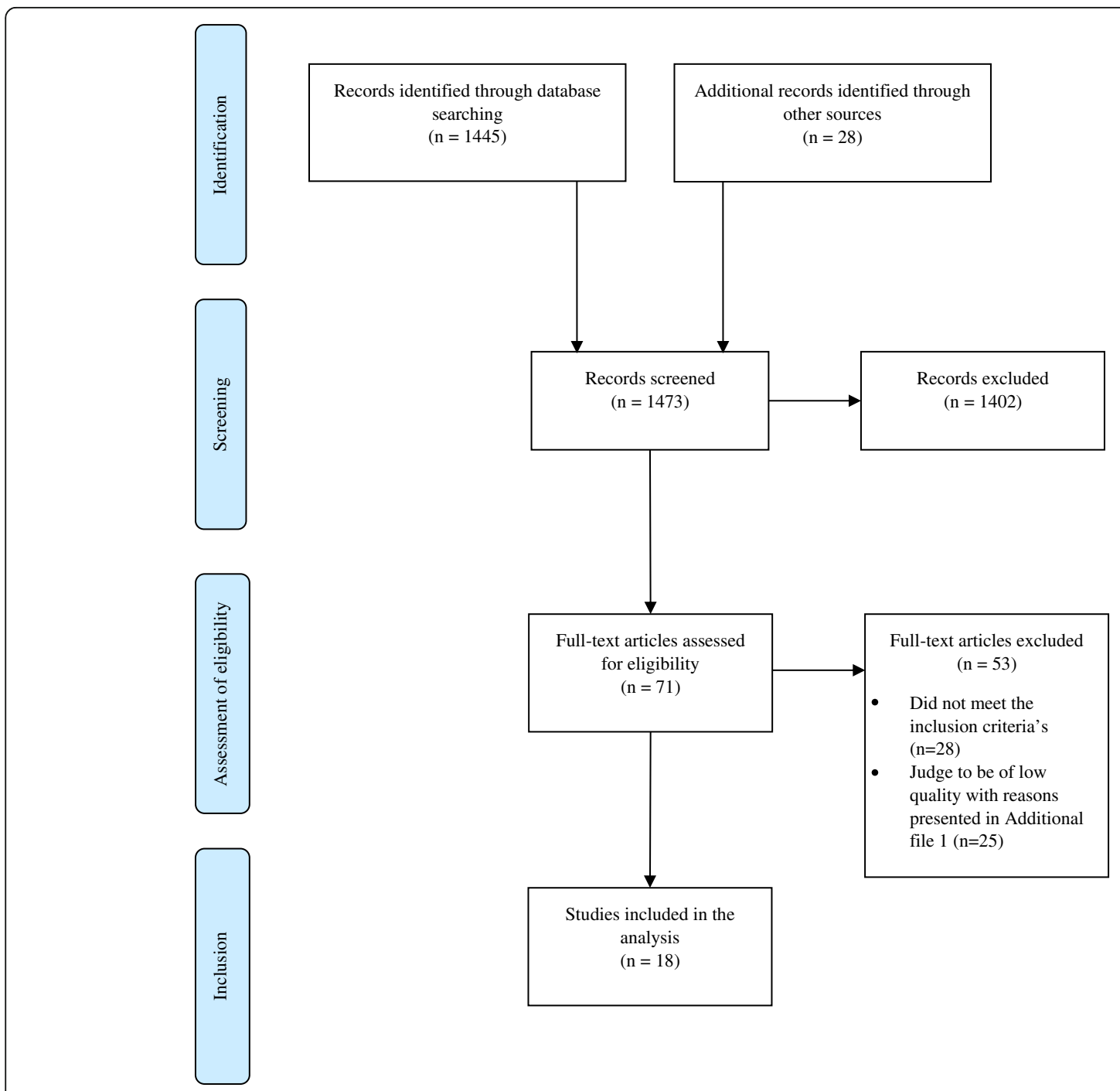

Fig. 2 Flow chart of the work process. PRISMA 2009 Flow Diagram [58] 


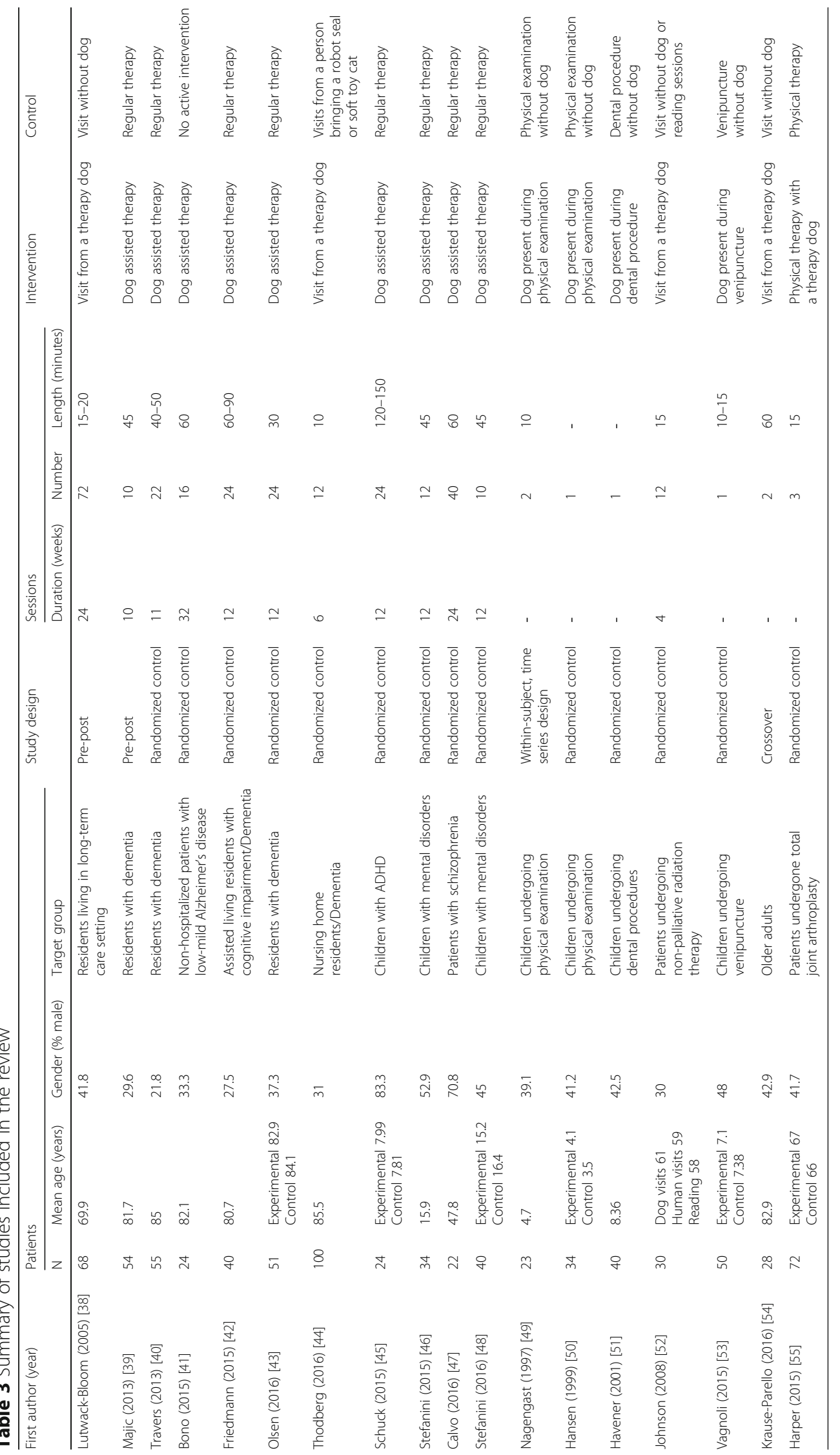




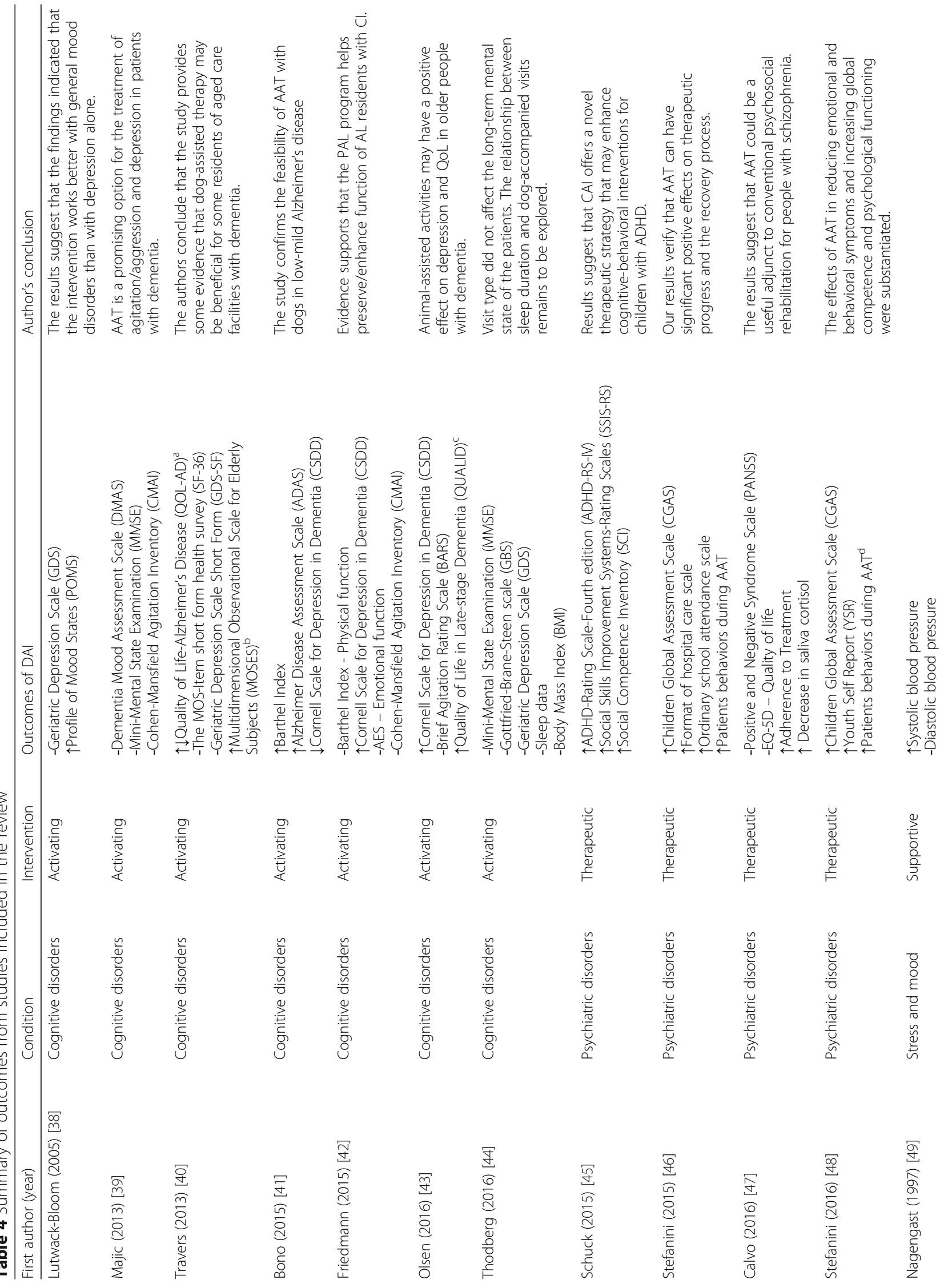


Lundqvist et al. BMC Complementary and Alternative Medicine (2017) 17:358

Page 7 of 12

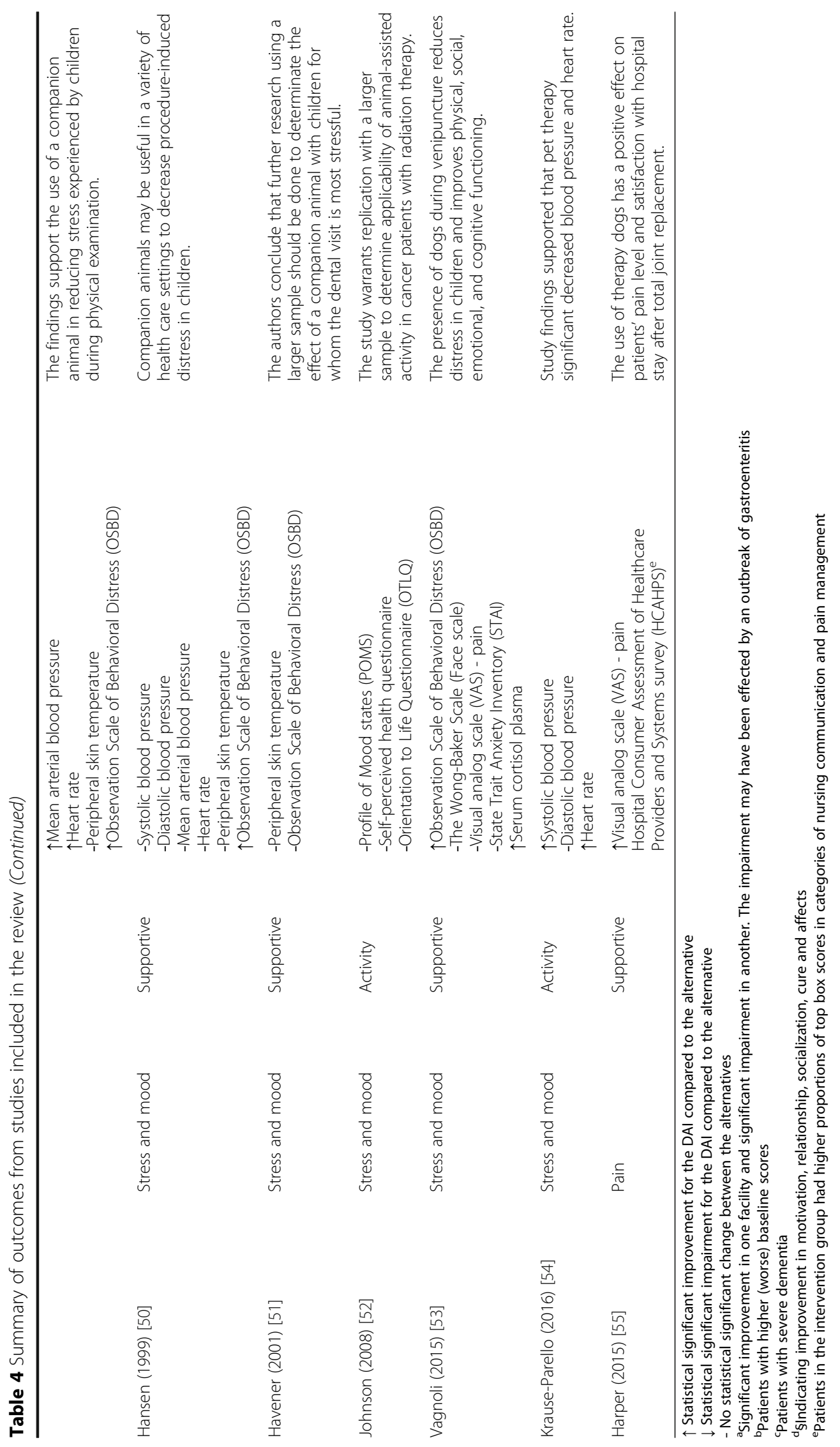


Table 5 Number of studies divided into condition, type of intervention, and the presence of positive outcome

\begin{tabular}{|c|c|c|c|c|c|c|}
\hline \multirow[t]{3}{*}{ Condition } & \multicolumn{2}{|c|}{ Therapeutic intervention } & \multicolumn{2}{|c|}{ Activating intervention } & \multicolumn{2}{|c|}{ Supportive intervention } \\
\hline & \multicolumn{6}{|c|}{ At least one significant positive outcome } \\
\hline & Yes & No & Yes & No & Yes & No \\
\hline Cognitive disorder & & & 6 & 1 & & \\
\hline Psychiatric disorder & 4 & & & & & \\
\hline Stress and mood & & & 1 & 1 & 3 & 1 \\
\hline Pain & & & & & 1 & \\
\hline
\end{tabular}

one study the intervention was preformed to reduce pain for patients undergoing total knee joint arthroplasty [55].

\section{Studied controls}

In all studies expect two, the control treatment was a visit, series of visits, a therapy session or series of therapy sessions without a dog. In the study by Thodberg et al. the controls were either a visit from a person bringing a robot seal or a visit from a person bringing a soft-toy cat [44]. In the study by Bono et al. the control group received no active intervention [41].

\section{Outcomes}

The studies included a variety of outcome measures and instruments (Table 4), physiological parameters e.g. blood pressure, heart rate, cortisol in saliva, skin temperature; disease specific measures e.g. dementia mood assessment scale, geriatric depression scale, Cohen-Mansfield agitation inventory; general functional measures e.g. observation scale of behavioral distress, profile of mood states, self-perceived health questionnaire, orientation to life questionnaire; generic health related quality of life measures e.g. EQ-5D, quality of life in late-stage dementia; and other measurements including e.g. sleep data, body mass index, and ordinary school attendance. In the column 'Outcomes of DAI' (Table 4) we have summarized the outcome changes for the different studies, based on statistical significance.

Table 5 shows the number of studies with at least one statistically significant positive outcome measure divided by patient condition and intervention category.

\section{Cognitive disorders}

Seven trials studied cognitive disorders and were all categorized as DAA [38-44]. They differed in terms of patient population severity, which varied from mild cognitive impairment to severe dementia. In all studies multiple sessions were used during periods varying in length from 6 to 32 weeks. The number of sessions varied between 12 and 72, and the length of sessions were 10-90 $\mathrm{min}$.

The studies also differed regarding control group treatments. In Friedman et al., Thodberg et al. and Lutwack et al. the control group participants were given another structured intervention [38, 42, 44]. In Majic et al., Olsen et al. and Travers et al. the control group was treated as usual [39, 40, 43]. In the study conducted by Bono et al. the control group received no active intervention [41].

Two of the studies showed some decrease in depression specific instruments $[40,42]$. Travers et al. showed that patients with worse baseline depression scores in the DAA group, significantly improved depression scores in comparison to the control group. They also found significant improvements in quality of life in one of the facilities studied, but these results were confounded by an outbreak of gastroenteritis with subsequent significant decrease in QoL in another unit [40]. In the Friedman et al. study, depression decreased significantly in the DAA group after three months but not in the control group [42]. In the study by Olsen et al. patients with severe dementia in the DAA group had an improved quality of life at follow up [43]. Bono et al. showed a significant difference in reduction of functional status between the DAA group and the control group after eight months. In the DAA group, the development of cognitive impairment also slowed up in comparison to the control group [41]. In Thodberg et al. the sessions lasted only $10 \mathrm{~min}$ and the control group were activated with a robot seal or a soft toy cat. They found no effect in measures of depression [44]. Also in Lutwack et al. the patients only received short sessions, the results showed no effect in measures of depression but a significant improvement in mood for those receiving visits from a therapy dog [38].

Concerning cognitive disorder, we conclude that the included studies differed in terms of severity of patient conditions, and in particular in the characteristics of DAA and the activity offered to the control group. Given these differences, treatment of cognitive disorders in a nursing home setting may result in some positive effects on health and wellbeing, most likely on depression and on quality of life for patients with severe dementia.

\section{Psychiatric disorders}

The four trials that studied psychiatric disorders were all categorized as DAT, and were randomized in a pre-post 
experimental design. Three of the included studies investigated patients in child and adolescent psychiatry $[45,46,48]$, and one studied patients from adult psychiatry [47]. All of the studies comprised 12 week DAT programs in different settings except for the study conducted by Calvo et al., where the intervention lasted 24 weeks [47].

In both studies conducted by Stefanini et al. DAT was compared with standard treatment in children and adolescents admitted to a psychiatry unit for acute mental disorder [46, 48], while Schuck et al. compared cognitive-behavioral intervention delivered with or without DAT in children with ADHD [45]. In the study by Calvo et al. [47] of adult inpatients with schizophrenia, DAT was assessed as an adjunct to, and in comparison with, conventional psychosocial rehabilitation.

All three studies of young patients with psychiatric disorder showed that DAT resulted in significant improvements on different psychometric scales and measures $[45,46,48]$. The two DAT studies, studying young patients with acute mental disorders, found improvements in global functioning, school attendance, as well as self-reported emotional-behavioral symptoms $[46,48]$. DAT in ADHD children resulted in a greater reduction in the severity of ADHD symptoms in comparison to cognitive-behavioral interventions without DAT [45]. Calvo et al. showed that after 24 weeks of rehabilitation the DAT group had no benefit on the Positive and Negative Syndrome Scale (PANSS), but in comparison with conventional rehabilitation, a significant reduction of negative symptomatology, a higher adherence to the program, and cortisol reduction were found after the DAT sessions [47].

In summary, all studies of DAT in psychiatric disorders showed significant reductions in symptoms, and higher program adherence.

\section{Stress and mood}

Seven trials studied stress and mood, five of them were categorized as DAS and two as DAA. Nagengast et al., Hansen et al. and Havener et al. examined effects of DAS on physiological and behavioral distress among children undergoing a physical examination or a dental procedure [50,51]. Vagnoli et al. studied the effects of DAS in children undergoing venipuncture [53]. Johnson et al. conducted a study in a radiation oncology unit examining effects of DAA among patients undergoing non-palliative radiation therapy [52]. Krause-Parello et al. [54] investigated changes in older adult's cardiovascular health before and after a DAA.

In the study by Nagengast et al. the children were exposed to two examinations, one with and one without a dog present [49]. In Hansen et al., Havener et al. and Vagnoli et al. the children underwent a procedure with or without the presence of a $\operatorname{dog}[50,51,53]$. In the study by Johnson et al. two control groups were constructed, one receiving visits from a human person and one where the patients read magazines [52]. In the study by Krause-Parello et al. all patients received two visits; one with and one without a dog [54].

In four of the six studies, the populations studied were children and the number of sessions was limited to one [49-51, 53]. Both Nagengast et al. and Hansen et al. found lower stress levels during physical examination when a dog was present, compared to not present, measured with the Observation Scale of Behavioral Distress (OSBD) [49, 50]. In addition, Nagengast et al. reported a statistically significant decrease in mean arterial and systolic blood pressure, heart rate and behavioral distress in the presence of a dog [49]. Vagnoli et al. also reported significant lower stress levels measured with OSBD, when a dog was present, in children undergoing venipuncture [53]. They also found significant lower levels of serum cortisol plasma in the intervention group compared with the control group [53]. The study conducted by Havener et al., examining children undergoing a dental procedure, was very similar to the other studies but showed no significant effects [51]. The study conducted by Johnson et al. differed from the other studies. They investigated the effects of DAA during a four-week period, including 12 sessions on adults undergoing non-palliative radiation therapy. They found no effects of DAA [52]. The study by Krause-Parello et al. also differed from the other studies [54]. The study was conducted on older adult patients living at home who did not have any specific condition. The results implied a greater decrease in systolic blood pressure when visited by a dog compared to a human person. From the results they also predicted more of a decrease in heart rate during the DAA compared with the conventional intervention [54].

In summary, four out of six studies showed at least one significant positive effect. Taken together, these findings suggest that particular DAS may reduce stress and positively affect the mood.

\section{Pain}

Harper et al. studied the role of DAS in postoperative recovery in patients after total joint arthroplasty [55]. Patients in the intervention group received a 15-min visit from a dog before the patient underwent physical therapy over a three-day period. The control group underwent physical therapy without any changes to normal routine. Patients in the intervention group reported a significant reduction of pain measured with the visual analog scale (VAS) compared with the control group [55]. Like the other studies categorized as supportive interventions reporting positive effects, the study included few and short sessions. 


\section{Discussion}

Even though there is a growing number of studies reported on use of animals in healthcare, the evidence base appears weak, partly due to studies including a limited number of participants. Previous reviews of AAI studies have focused exclusively on a single condition or a specific population. This review was delimited to dogs used as the assisting animal in a health care setting but without any limitation of the characteristics of the population. The studies fulfilling our selection criteria included diverse conditions and outcome measures. The findings were not consistent, and studies of costeffectiveness were lacking.

Substantial differences were observed in the manner in which DAI was applied. Three major categories of interventions were identified; those used for therapeutic purpose, those used for activating purpose and those used as support during a procedure. Even if we were unable to draw firm conclusions regarding specific applications from the studies included in the current review, some promising results were seen. DAT seemed to be most substantial in treatment of psychiatric disorders both among young and adult patients. DAA appeared to have some positive effects on health and wellbeing as well as an effect on depression and quality of life in severe cognitive disorders. We also found that DAS may have positive effects on stress and mood.

Previous systematic reviews of the scientific literature regarding the use of DAI in health care settings showed some evidence of patient benefit. However, many reviews included a mix of animals and only a handful focused exclusively on DAI. One review of therapy dogs for children with autism spectrum disorders, included four studies on DAI in health care. It concluded that the results were encouraging but further research with better designs and larger samples were needed [56]. Another review of AAT in treatment of similar patient material included fourteen studies with different kinds of animals. Seven of these studies involved dogs and showed positive outcomes. However, most studies included were connected with methodological weaknesses [8]. The evidence base seemed to be better for the use of DAI for residents of long-term care facilities. Cipriani et al. showed that 12 of 19 studies reported statistically significant outcomes [57]. Even though there is some systematic, and a substantial amount of anecdotal evidence for the effects of animals as interventions in health care, the evidence base seems to be relatively weak.

Overall, in all the included studies, DAIs were offered in combination with regular medical treatment, which means that the control group always received regular treatment. In general though, we found that the included studies were fraught with numerous obstacles and confounding factors. For example, lack of proper control group and lack of a proper control activity, were common. Also, most studies had not attempted to investigate the impact of the dog handler. Besides that, there were natural difficulties in randomization due to patient preferences, fears and possible allergies. Given all the challenges mentioned above, it was a matter of concern that the number of participants was generally too small to minimize the effects of the multitude of lack of control and confounding factors involved. It is possible, and even likely, that matters of suboptimal study designs may explain some of the observed differences in the effects of DAI. It is also likely that the limited number of subjects included in most of the studies explained the non-statistically significant effects (type II statistical error). It is also possible that the instruments used in the studies for measuring treatment effects did not optimally measure the effect of all important aspects of increased wellbeing. Studies of DAI is evidently challenging regarding research design, since none of the studies we selected fulfilled our criteria of "high quality".

Only 18 studies were included in the review as a primary basis for our conclusions. This limited number may be due to the strict criteria applied by us, to poor study designs, or both. One weakness with our review may be that we excluded studies with less than 20 participants. It is possible that there exist studies based on small samples that could be judged to have at least moderate quality. There is also a possibility that we have wrongly classified one or several studies, but that would hardly affect our conclusion. Another weakness of a review linked to inclusion criteria, is the limitation of including only published articles from peer-reviewed journals with full text in English. This is a common delimitation due to a lack of resources, but of course a weakness.

\section{Conclusion}

The overall assessment of the included studies indicates minor to moderate effects of dog-assisted therapy in psychiatric conditions, as well as for dog-assisted activities in cognitive disorders and for dog-assisted support in different types of medical interventions. However, the majority of studied outcome measures showed no significant effect.

\section{Additional file}

Additional file 1: Excluded studies due to low quality with reasons for exclusions. (DOCX $17.6 \mathrm{~kb}$ )

\section{Abbreviations}

AAA: Animal-assisted activity; AAE: Animal-assisted education; AAl: Animalassisted intervention; AAT: Animal-assisted therapy; ADAS: Alzheimer Disease Assessment Scale; ADHD-RS-IV: ADHD-Rating Scale-Fourth edition; BARS: Brief Agitation Rating Scale; BMl: Body Mass Index; C-GAS: Children Global Assessment Scale; CMAI: Cohen-Mansfield Agitation Inventory; CSDD: Cornell 
Scale for Depression in Dementia; DAA: Dog-assisted activity; DAl: Dog-assisted intervention; DAS: Dog-assisted support; DAT: Dog-assisted therapy; DMAS: Dementia Mood Assessment Scale; GBS: Gottfried-BraneSteen scale; GDS: Geriatric Depression Scale; HCAHPS: Hospital Consumer Assessment of Healthcare Providers and Systems survey; MMSE: Mini-Mental State Examination; MOSES: Multidimensional Observational Scale for Elderly Subjects; OSBD: Observation Scale of Behavioral Distress; OTLQ: Orientation to Life Questionnaire; PANSS: Positive and Negative Syndrome Scale; POMS: Profile of Mood States; QOL-AD: Quality of Life-Alzheimer's Disease; QUALID: Quality of Life in Late-stage Dementia; SCI: Social Competence Inventory; SSIS-RS: Social Skills Improvement Systems-Rating Scales; STAI: State Trait Anxiety Inventory; VAS: Visual Analog Scale; YSR: Youth Self Report

\section{Acknowledgements}

None.

\section{Funding}

This study was funded by Region Östergötland, Sweden.

\section{Availability of data and materials}

The dataset supporting the conclusions of this article is included within the article.

\section{Authors' contributions}

$M L, P C, E T, R S$ and $L A ̊ L$ designed the study. ML conducted the literature search. In collaboration ML, PC, ET, RS and LÅL analyzed and interpreted the data. ML, PC, ET, RS, LÅL contributed to the drafting of the manuscript. All authors critical revised and approved the final manuscript.

\section{Ethics approval and consent to participate}

Not applicable.

\section{Consent for publication}

Not applicable.

\section{Competing interests}

The authors declare that they have no competing interests.

\section{Publisher's Note}

Springer Nature remains neutral with regard to jurisdictional claims in published maps and institutional affiliations.

\section{Author details}

${ }^{1}$ Department of Medical and Health Sciences, Division of Health Care Analysis, Linköping University, 581 83, Linköping, Sweden. Department of Clinical and Experimental Medicine, Linkoping University, Linköping, Sweden. ${ }^{3}$ Department of Clinical Chemistry and Department of Clinical and Experimental Medicine, Linköping University, Linköping, Sweden.

Received: 11 April 2017 Accepted: 19 June 2017

Published online: 10 July 2017

\section{References}

1. Fine $\mathrm{AH}$. Handbook on animal-assisted therapy: foundations and guidelines for animal-assisted interventions. 4th ed. San Diego, CA, US: Elsevier Academic Press; 2015.

2. Pet Partners. Terminology. https://petpartners.org/learn/terminology/. Accessed 17 Feb 2017

3. International Association of Human-Animal Interaction Organizations (IAHAIO). IAHAIO White Paper 2014 - The IAHAIO definitions for animal assisted intervention and animal assisted activity and guidelines for wellness of animals involved. 2014. http://www.iahaio.org/new/fileuploads/ 4163IAHAIO\%20WHITE\%20PAPER-\%20FINAL\%20-\%20NOV\%2024-2014.pdf. Accessed 17 Feb 2017

4. American Veterinary Medical Association (AVMA). Animal-Assisted Interventions: Definitions. https://www.avma.org/KB/Policies/Pages/AnimalAssisted-Interventions-Definitions.aspx. Accessed 17 Feb 2017.

5. Custance D, Mayer J. Empathic-like responding by domestic dogs (Canis familiaris) to distress in humans: an exploratory study. Anim Cogn. 2012;15(5):851-9.
6. Kamioka H, Okada S, Tsutani K, Park H, Okuizumi H, Handa S, et al. Effectiveness of animal-assisted therapy: a systematic review of randomized controlled trials. Complement Ther Med. 2014;22(2):371-90.

7. Maber-Aleksandrowicz S, Avent C, Hassiotis A. A Systematic Review of Animal-Assisted Therapy on Psychosocial Outcomes in People with Intellectual Disability. Res Dev Disabil. 2016;49-50:322-38.

8. O'Haire ME. Animal-assisted intervention for autism spectrum disorder: a systematic literature review. J Autism Dev Disord. 2013;43(7):1606-22.

9. Nimer J, Lundahl B. Animal-assisted therapy: a meta-analysis. Anthrozoös. 2007:20(3):225-38.

10. Muñoz LS, Máximo BN, Valero AR, Atín AMA, Varela DE. Ferrieroc G. Animal assisted interventions in neurorehabilitation: a review of the most recent literature Neurologia. 2015;30(1):1-7.

11. Egger M, Smith GD, Altman DG. Systematic reviews in health care: metaanalysis in context. 2nd ed. London: BMJ Books; 2001.

12. Riegelman R. Studying a Study \& Testing a test: reading evidence-based Health Research. 6th ed. United States, North America: Health Sciences Research Commons; 2012.

13. Crowley-Robinson P, Fenwick DC, Blackshaw JK. A long-term study of elderly people in nursing homes with visiting and resident dogs. Appl Anim Behav Sci. 1996:47(1-2):137-48.

14. Zisselman MH, Rovner BW, Shmuely Y, Ferrie P. A pet therapy intervention with geriatric psychiatry inpatients. Am J Occup Ther. 1996:50(1):47-51.

15. Barker SB, Dawson KS. The effects of animal-assisted therapy on anxiety ratings of hospitalized psychiatric patients. Psychiatr Serv. 1998;49(6):797-801.

16. Barker SB, Pandurangi AK, Best AM. Effects of animal-assisted therapy on patients' anxiety, fear, and depression before ECT. J ect. 2003;19(1):38-44.

17. Richeson NE. Effects of animal-assisted therapy on agitated behaviors and social interactions of older adults with dementia: an evidence-based therapeutic recreation intervention. Am J of Recreation Ther. 2003;2(4):9-16 8p.

18. Nathans-Barel I, Feldman P, Berger B, Modai I, Silver H. Animal-assisted therapy ameliorates anhedonia in schizophrenia patients. A controlled pilot study. Psychother Psychosom. 2005;74(1):31-5

19. Prothmann A, Bienert M, Ettrich C. Dogs in child psychotherapy: effects on state of mind. Anthrozoös. 2006;19(3):265-77.

20. Cole KM, Gawlinski A, Steers N, Kotlerman J. Animal-assisted therapy in patients hospitalized with heart failure. Am J Crit Care. 2007;16(6):575-85.

21. Orlandi M, Trangeled K, Mambrini A, Tagliani M, Ferrarini A, Zanetti L, et al. Pet therapy effects on oncological day hospital patients undergoing chemotherapy treatment. Anticancer Res. 2007:27(6c):4301-3.

22. Banks MR, Willoughby LM, Banks WA. Original study: animal-assisted therapy and loneliness in nursing homes: use of robotic versus living dogs. J Am Med Dir Assoc. 2008;9:173-7

23. Martindale BP. Effect of animal-assisted therapy on engagement of rural nursing home residents. American Journal of Recreation Therapy. 2008;7(4):45-53.

24. Braun C, Stangler T, Narveson J, Pettingell S. Animal-assisted therapy as a pain relief intervention for children. Complement Ther Clin Pract. 2009;15(2):105-9.

25. Chu Cl, Liu CY, Sun CT, Lin J. The effect of animal-assisted activity on inpatients with schizophrenia. J Psychosoc Nurs Ment Health Serv. 2009; 47(12):42-8

26. Villalta-Gil V, Roca M, Gonzalez N, Domenec E. Cuca, Escanilla a, Asensio MR, Esteban ME, Ochoa S, Haro JM. Dog-assisted therapy in the treatment of chronic schizophrenia inpatients. Anthrozoös. 2009;22(2):149-59.

27. Moretti F, De Ronchi D, Bernabei V, Marchetti L, Ferrari B, Forlani C, et al. Pet therapy in elderly patients with mental illness. Psychogeriatrics. 2011;11(2):125-9.

28. Beck CE, Gonzales F Jr, Sells CH, Jones C, Reer T, Zhu YY. The effects of animal-assisted therapy on wounded warriors in an Occupational therapy life Skills program. US Army Med Dep J. 2012:38-45.

29. Dietz TJ, Davis D, Pennings J. Evaluating animal-assisted therapy in group treatment for child sexual abuse. J Child Sex Abus. 2012;21(6):665-83.

30. Marcus DA, Bernstein CD, Constantin JM, Kunkel FA, Breuer P, Hanlon RB. Animal-assisted therapy at an outpatient pain management clinic. Pain Med. 2012;13(1):45-57

31. Marcus DA, Bernstein CD, Constantin JM, Kunkel FA, Breuer P, Hanlon RB. Impact of animal-assisted therapy for outpatients with fibromyalgia. Pain Med. 2013:14(1):43-51.

32. Vrbanac Z, Zecevic I, Ljubic M, Belic M, Stanin D, Bottegaro NB, et al. Animal assisted therapy and perception of loneliness in geriatric nursing home residents. Coll Antropol. 2013;37(3):973-6. 
33. Havey J, Vlasses FR, Vlasses PH, Ludwig-Beymer P, Hackbarth D. The effect of animal-assisted therapy on pain medication use after joint replacement. Anthrozoös. 2014;27(3):361-9.

34. Nordgren L, Engstrom G. Effects of dog-assisted intervention on behavioural and psychological symptoms of dementia. Nurs Older People. 2014;26(3):31-8.

35. Barker SB, Knisely JS, Schubert CM, Green JD, Ameringer S. The effect of an animal-assisted intervention on anxiety and pain in hospitalized children. Anthrozoös. 2015;28(1):101-12.

36. Calcaterra V, Veggiotti $P$, Palestrini C, De Giorgis V, Raschetti $R$, Tumminelli $\mathrm{M}$, et al. Post-operative benefits of animal-assisted therapy in pediatric surgery: a randomised study. PLoS One. 2015;10(6):e0125813.

37. Nurenberg JR, Schleifer SJ, Shaffer TM, Yellin M, Desai PJ, Amin R, et al. Animal-assisted therapy with chronic psychiatric inpatients: equine-assisted psychotherapy and aggressive behavior. Psychiatr Serv. 2015;66(1):80-6.

38. Lutwack-Bloom P, Wijewickrama R, Smith B. Effects of pets versus people visits with nursing home residents. J Gerontol Soc Work. 2005;44(3/4):137-59.

39. Majić T, Rapp MA, Gutzmann H, Heinz A, Lang UE. Animal-assisted therapy and agitation and depression in nursing home residents with dementia: a matched caseecontrol trial. Am J Geriatr Psychiatr. 2013;21(11):1052-9.

40. Travers C, Perkins J, Rand J, Bartlett H, Morton J. An evaluation of dogassisted therapy for residents of aged care facilities with dementia. Anthrozoös. 2013;26(2):213-25.

41. Bono AV, Benvenuti C, Buzzi M, Ciatti R, Chiarelli V, Chiambretto P, et al. Effects of animal assisted therapy (AAT) carried out with dogs on the evolution of mild cognitive impairment. G Gerontol. 2015;63(1):32-6.

42. Friedmann E, Galik E, Thomas SA, Hall PS, Chung SY, McCune S. Evaluation of a pet-assisted living intervention for improving functional status in assisted living residents with mild to moderate cognitive impairment: a pilot study. Am J Alzheimers Dis Other Demen. 2015;30(3):276-89.

43. Olsen C, Pedersen I, Bergland A, Enders-Slegers MJ, Patil G, Ihlebaek C. Effect of animal-assisted interventions on depression, agitation and quality of life in nursing home residents suffering from cognitive impairment or dementia: a cluster randomized controlled trial. Int J Geriatr Psychiatry. 2016; 31(12):1312-21.

44. Thodberg K, Sorensen LU, Christensen JW, Poulsen PH, Houbak B, Damgaard V, et al. Therapeutic effects of dog visits in nursing homes for the elderly. Psychogeriatrics. 2016;16(5):289-97.

45. Schuck SE, Emmerson NA, Fine AH, Lakes KD. Canine-assisted therapy for children with ADHD: preliminary findings from the positive assertive cooperative kids study. J Atten Disord. 2015;19(2):125-37.

46. Stefanini MC, Martino A, Allori P, Galeotti F, Tani F. The use of animalassisted therapy in adolescents with acute mental disorders: a randomized controlled study. Complement Ther Clin Pract. 2015;21(1):42-6.

47. Calvo P, Fortuny JR, Guzman S, Macias C, Bowen J, Garcia ML, et al. Animal assisted therapy (AAT) program as a useful adjunct to conventional psychosocial rehabilitation for patients with schizophrenia: results of a small-scale randomized controlled trial. Front Psychol. 2016;7:631.

48. Stefanini MC, Martino A, Bacci B, Tani F. The effect of animal-assisted therapy on emotional and behavioral symptoms in children and adolescents hospitalized for acute mental disorders. European Journal of Integrative Medicine. 2016;8(2):81-8.

49. Nagengast SL, Baun MM, Megel M, Leibowitz JM. The effects of the presence of a companion animal on physiological arousal and behavioral distress in children during a physical examination. J Pediatr Nurs. 1997:12(6):323-30

50. Hansen KM, Messinger CJ, Baun MM, Megel M. Companion animals alleviating distress in children. Anthrozoös. 1999;12(3):142-8.

51. Havener L, Gentes L, Thaler B, Megel ME, Baun MM, Driscoll FA, et al. The effects of a companion animal on distress in children undergoing dental procedures. Issues Compr Pediatr Nurs. 2001;24(2):137-52.

52. Johnson RA, Meadows RL, Haubner JS, Sevedge K. Animal-assisted activity among patients with cancer: effects on mood, fatigue, self-perceived health, and sense of coherence. Oncol Nurs Forum. 2008;35(2):225-32.

53. Vagnoli L, Caprilli S, Vernucci C, Zagni S, Mugnai F, Messeri A. Can presence of a dog reduce pain and distress in children during venipuncture? Pain Manag Nurs. 2015;16(2):89-95.

54. Krause-Parello CA, Kolassa J. Pet therapy: enhancing social and cardiovascular wellness in community dwelling older adults. J Community Health Nurs. 2016;33(1):1-10.
55. Harper CM, Dong Y, Thornhill TS, Wright J, Ready J, Brick GW, et al. Can therapy dogs improve pain and satisfaction after total joint arthroplasty? A randomized controlled trial. Clin Orthop Relat Res. 2015;473(1):372-9.

56. Berry A, Borgi M, Francia N, Alleva E, Cirulli F. Use of assistance and therapy dogs for children with autism Spectrum disorders: a critical review of the current evidence. Journal of Alternative \& Complementary Medicine. 2013. 19(2):73-80

57. Cipriani J, Cooper M, DiGiovanni NM, Litchkofski A, Nichols AL, Ramsey A. Dog-assisted therapy for residents of long-term care facilities: an evidencebased review with implications for Occupational therapy. Physical \& Occupational Therapy in Geriatrics. 2013;31(3):214-40.

58. Moher D, Liberati A, Tetzlaff J, Altman DG. Preferred reporting items for systematic reviews and meta-analyses: the PRISMA statement. Ann Intern Med. 2009;151(4):264-9.

\section{Submit your next manuscript to BioMed Central and we will help you at every step:}

- We accept pre-submission inquiries

- Our selector tool helps you to find the most relevant journal

- We provide round the clock customer support

- Convenient online submission

- Thorough peer review

- Inclusion in PubMed and all major indexing services

- Maximum visibility for your research

Submit your manuscript at www.biomedcentral.com/submit
) Biomed Central 